\title{
Analytical modelling of high velocity impacts of cylindrical projectiles on carbon/epoxy laminates
}

\author{
J. López-Puente*, D. Varas, J.A. Loya, R. Zaera \\ Department of Continuum Mechanics and Structural Analysis, University Carlos III of Madrid, Avda. de la Universidad, 30, 28911 Leganés, Madrid, Spain
}

\begin{abstract}
A B S T R A C T
In this work an analytical model has been developed in order to predict the residual velocity of a cylin drical steel projectile, after impacting into a woven carbon/epoxy thin laminate. The model is based in an energy balance, in which the kinetic projectile energy is absorbed by the laminate through three different mechanisms: linear momentum transfer, fiber failure and laminate crushing. This last mechanism needs the quantification of the through thickness compressive strength, which has been evaluated by means of quasi static punch tests. Finally, high velocity impact tests have been accomplished in a wide range of velocities, to validate the model.
\end{abstract}

\section{Introduction}

Laminated composite structures are attracting the interest of a number of leading aircraft and spacecraft engineering manufactur ers, due to their high strength to weight and stiffness to weight ratios and to the flexible tailoring of their properties in a required direction. The most used composite materials for structural appli cations in these sectors are Carbon Fiber Reinforced Plastics (CFRP), commonly manufactured with an epoxy matrix, which combines good mechanical properties, high resistance to corrosion and fati gue, and low density $\left(\rho=1500 \mathrm{~kg} / \mathrm{m}^{3}\right)$. CFRP applications in com mercial aircraft have been steadily increasing [1] as raw material costs are reduced, automation of manufacturing processes evolves and experience in design technology is increased. At least $50 \%$ of the next generation of military and civil aircraft structures are likely to be made mainly from composites [2].

Vulnerability against high velocity impact loads is becoming an increasingly critical issue for the design of CFRP aerospace struc tures in recent years. Bird strikes [3] and hailstones [4] are the most critical situations because of its high probability of occur rence and its disastrous consequences, especially when ingested by the engine. Also the ice released from the edge of a propeller blade may impact the nacelle of the twin engine or the fuselage, and runway debris may impact the underside of wing structures [5]. The Concorde accident in 2000 was actually caused by a tire fragment impacting a fuel tank in the wing of the airplane. Such an impact results in a pressure wave that propagates through the

* Corresponding author.

E-mail address: jlpuente@ing.uc3m.es (J. López-Puente). fluid within the tank, stresses the walls, and increases the risk of catastrophic wing failure [6,7]. Another catastrophic example was the impact of frozen lightweight foam projectiles on the wing of the Space Shuttle. Other kinds of projectiles that may impact air craft structures are small and medium caliber bullets and frag ments due to a blast, both of them causing hydrodynamic ram effects when impacting the fuel tank of an airplane or a helicopter. The aeroengine turbine blade may also fail due to fatigue and may penetrate the wall of the containment cell, damaging oil tanks and airframes [8]. Impact engineering is also of great interest in the field of spacecraft, even if the failure mechanisms are considerably different in this hyper velocity range. The near earth space envi ronment, where satellites, the International Space Station, and the Space Shuttle orbit the earth, is jumbled with both artificial and natural debris $[9,10]$. This debris is too small and numerous to be individually tracked and there are many satellites in orbit, so that the average time between destructive collisions is about 10 years. CFRPs are well known to be particularly vulnerable to im pact of foreign objects, especially because of the brittleness of the polymeric phase, giving rise to a multiplicity of failure modes and leading to significant strength reduction in post damage perfor mance. Therefore understanding its response to a range of poten tial impact loadings and resulting damage mechanisms is essential for the successful use of these materials.

The response of CFRP panels to low or to high velocity impacts is quite different [11]. The response of CFRPs to low velocity impact damage few meters per second has been widely studied, using experimental test methods such as pendulum Charpy or drop tower. However, the number of papers concerned with the impact response of carbon fiber laminates under impact at hundreds of 
meters per second is relatively small. Experimental results of steel projectiles impacting CFRPs at high speed velocities can be found in papers due to Cantwell and Morton [11], Sun and Potti [12], Larsson [13], Bland and Dear [14], López Puente et al. [15,16], Will et al. [17], Tanabe et al. [18,19], Hammond et al. [20], Hosur et al. [21], Herzsberg and Weller [22], Caprino et al. [23] and Hazell et al. [24]. Other authors used projectiles made of different materials: Kim et al. [25] utilized ice spheres, Chambers et al. [26] lead pellets and Asp and Juntikka [27] ice spheres and granite stones.

These authors described the impact of a projectile onto the composite plate in different phases: initial contact and stress wave propagation, compression and local punch, plug formation under shear and compression, fiber breakage at the rear layers, and final perforation. During the penetration process the initial energy of the projectile is totally (in case of arrest) or partially (in case of per foration) absorbed by the laminate by kinetic energy, strain energy or damage mechanisms, namely matrix cracking, delamination, fi ber shear, or tensile fiber failure. In high velocity impacts, the main feature of these energy dissipation phenomena is the local re sponse of the material, because of the small mass which impacts and the short contact duration so that available kinetic energy of the projectile is likely distributed over a small volume surrounding the contact point.

The behavior of the composite laminates is influenced by a number of parameters including fiber and matrix types, stacking sequence [17], woven or tape architecture [15], stitching [13,21], service temperature [15], impact velocity and angle [11,16] and shape of the impactor [28]. Therefore, quite complex numerical or analytical simulation models have been developed by several authors to predict the entire impact process. Beyond the predictive interest of these tools, simulation models can aid in the detailed understanding of the behavior of a CFRP laminate to a range of threats, providing detailed information about the penetration loads, absorbed energies and the damage evolution processes.

Numerical codes solve the variational equations of the Mechanics of Continuous Media by means of finite element, fi nite difference or meshless methods. Some works on numerical simulation of ballistic impacts in CFRPs appear in $[29,30]$. Although less expensive than experimental testing, they also en tail a high cost because of the price of the codes and the long computational time. On the tout of a low cost tool that would en able an impact problem to be solved in the shortest possible time and that would be easy to use, Fernández Fdz et al. [31] proposed a methodology based on artificial neural networks to study the high velocity impact of spheres into CFRP laminates. This tool showed a great handling simplicity as well as low computational cost. For a quick prediction of the performance of the laminate, analytical models have been also proposed $[11,23,32]$. These are derived from a qualitative understanding of the macroscopic phe nomena. The main drawback of this methodology is the limited applicability of a given model to a specific range of impact condi tions. However, the simplicity of these models make them useful at early stages of design, allowing a quick evaluation of the impact response.

This paper proposes an analytical model to predict the residual velocity of a cylindrical projectile after impacting at ballistic veloc ity regime against carbon/epoxy plain woven laminates. This sim ulation tool is formulated from an energy balance. To validate the proposed model, impacts at velocities between 100 and $400 \mathrm{~m} / \mathrm{s}$ were performed and the residual velocity of the projectile was measured. The model predicts with accuracy the residual velocity and estimates the minimum impact velocity of the projectile needed to perforate the laminate. Moreover, its formulation in terms of non dimensional parameters facilitates an analysis of influence of the different energy absorption mechanisms during the impact process.

\section{Model description}

The analytical model developed in this work is formulated from an energy balance. In materials such as carbon/epoxy woven lam inates, it is commonly considered that the kinetic energy of the projectile $E_{k}$ is absorbed by three different mechanisms: linear momentum transferred from the projectile to the detached part of laminate $E_{m}$, tensile fiber failure breakage $E_{f}$ and laminate break age due to crushing $E_{c}$ [32]. The delamination failure mechanism does not contribute noticeably to the energy absorption due to the low specific fracture energy of epoxy matrices; in addition woven laminates do not use to present this kind of damage when all the plies have the same orientation [16]. Consequently this failure mechanism has not been considered in the energy balance. The contribution of elastic deformation is usually neglected when compared with the other energy absorption processes. It is assumed that there is no plastic deformation of the steel projectile, as observed in this and previous works [15]. The following energy balance constitutes the fundamental equation for the analytical model:

$$
d E_{k} \quad d E_{c}+d E_{m}+d E_{f}
$$

A spatial rather than temporal integration will be chosen be cause the expressions get considerably simplified; using a tempo ral integration, an analytical resolution of the equations will not be possible. The terms that appear in Eq. (1) could be expressed as follows:

- Kinetic energy of the projectile: the variable $x$ represents the pro jectile position from the impact side of the composite laminate (Fig. 1). When the projectile moves from $x$ to $x+d x$, it loses a quantity of energy given by:

$d E_{k}(x) \quad \frac{1}{2} m_{p} d\left(v^{2}(x)\right)$

where $m_{p}$ is the projectile mass, that remains constant along the process, and $v$ its velocity, which varies with the position. This energy is absorbed by the laminate through the following mechanisms:

- Energy absorbed by linear momentum transfer: the differential laminate volume $A d x$ detached from the laminate is accelerated from the rest to the current projectile velocity, and it is assumed to remain attached to the projectile during the penetration. The associated amount of energy is written as:

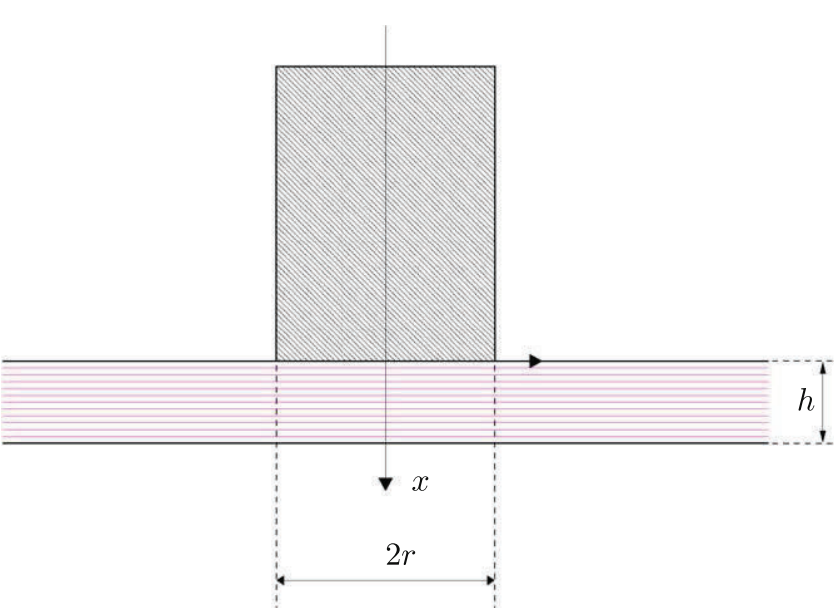

Fig. 1. Projectile and target configuration for zero projectile displacement. 


$$
d E_{m}(x) \quad \frac{1}{2}\left(\operatorname{Adx} \rho_{l}\right) v^{2}(x)
$$

where $\rho_{l}$ is the laminate density (the term in brackets represents the accelerated mass). The energy absorption mechanism disap pears when the penetration process finishes, when $x>h, h$ being the laminate thickness. Fig. 2 shows a sketch of this process.

- Energy absorbed by tensile fiber failure: this mechanism becomes relevant mainly at velocities close to those required to perforate the laminate (ballistic limit). In this work a 10 plies carbon/ epoxy woven laminate $\left([0]_{10}\right)$ was analyzed, with a total thick ness of $2.2 \mathrm{~mm}$. Fig. 3 shows an image of the transverse plane of a laminate impacted at $110 \mathrm{~m} / \mathrm{s}$, which is a velocity slightly below the ballistic limit. In the first plies a crushing breakage mechanism is observed, but the rest of the laminate fails due to tensile failure in the direction of the fibers. This last zone finally collapsed, creating roughly a $65^{\circ}$ truncated pyramidal volume [11], similar to that observed in brittle materials (such as ceramics) impacted transversally.

According to the analysis due to López Puente et al. [30,32], it was found that the time to tensile failure is equal to that needed by the elastic wave to reach the lower face of the laminate and re verse through the total thickness of the plate:

$t_{0} \frac{2 h}{\sqrt{ } E_{3} / \rho_{l}}$

$E_{3}$ being the through thickness elastic modulus. The differential en ergy associated with this failure mechanism is written as:

$d E_{f}(x) \quad \omega_{f} d V$

where $\omega_{f}$ is the specific energy and $d V$ the affected material volume at any $d x$. Assuming that the fiber behavior is elastic up to failure, the specific energy was estimated as:

$\omega_{f} \quad 2\left(\frac{1}{2} X_{t} \varepsilon_{f}\right)$

where $X_{t}$ is the tensile strength in direction 1 (and also in 2 on ac count of the woven configuration) and $\varepsilon_{f}$ is the ultimate strain. The term is multiplied by two, given the biaxial shape of the tensional field.

In order to define the affected volume $d V$, an analysis of the transverse section is done. Fig. 4 indicates that this absorption mechanism is located in a truncated pyramid, with an angle $\alpha$ close to $65^{\circ}$, and its diagonals are aligned in the fiber direction. The upper base semidiagonal is estimated to be equal to the pro jectile radius $r$, an the lower base semidiagonal is

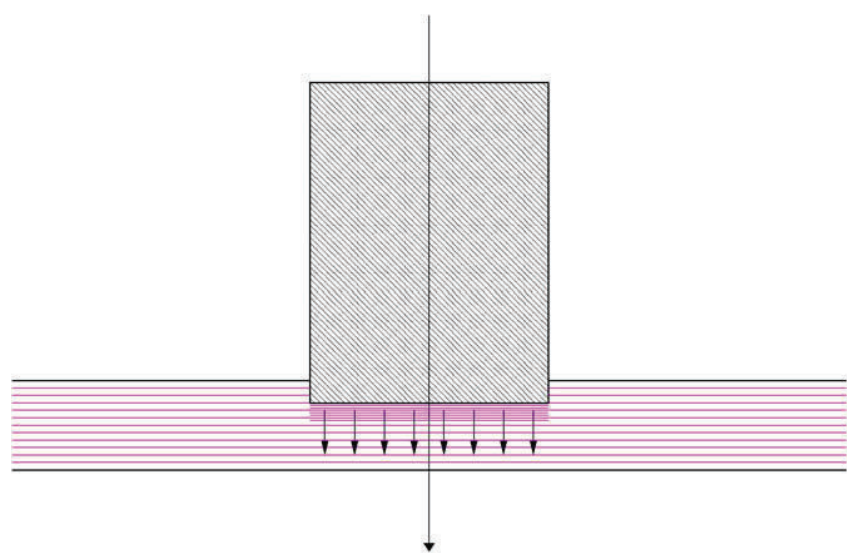

Fig. 2. Sketch of the linear momentum transfer process.

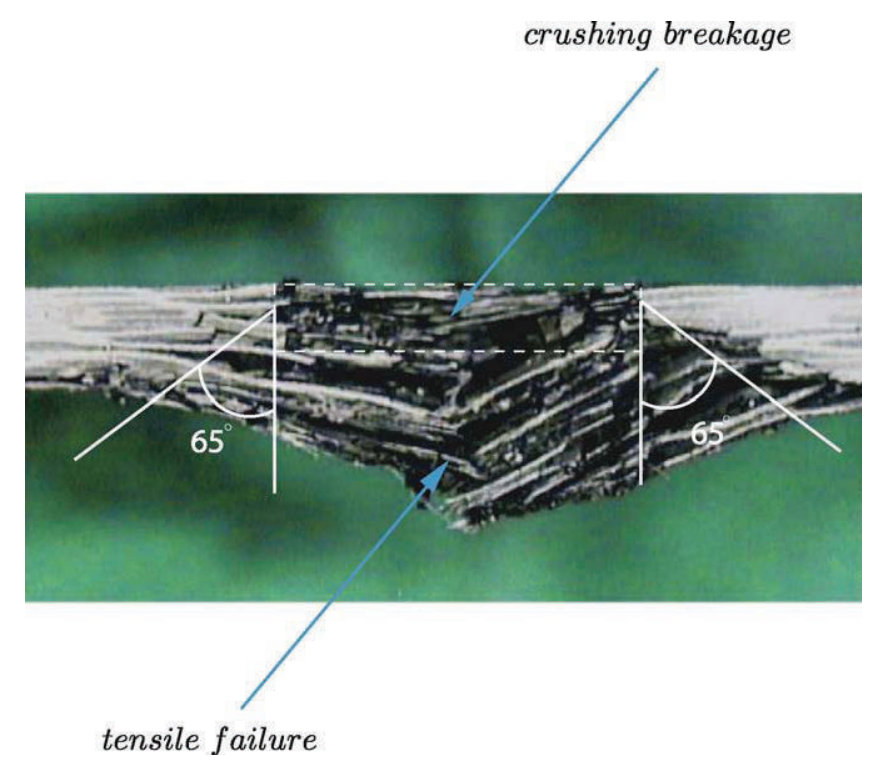

Fig. 3. Cross-section view of laminate impacted at $110 \mathrm{~m} / \mathrm{s}$.

$L \quad r+\left(\begin{array}{ll}h & x_{0}\end{array}\right) \tan \alpha$, with $x_{0}$ being the position of the projectile at $t \quad t_{0}$ (Fig. 4). Hence the affected volume for any $d x$ is calculated as:

$d V \quad 2 l^{2} d x \quad 2 d x\left(r+\left(\begin{array}{ll}x & x_{0}\end{array}\right) \tan \alpha\right)^{2}$

and the energy absorbed by this mechanism is:

$d E_{f} \quad X_{t} \varepsilon_{f} 2 d x\left(r+\left(x \quad x_{0}\right) \tan \alpha\right)^{2}$

Since this term participates in the impact process exclusively under certain conditions, it must be multiplied by a cutdown func tion $c_{r}(x)$ :

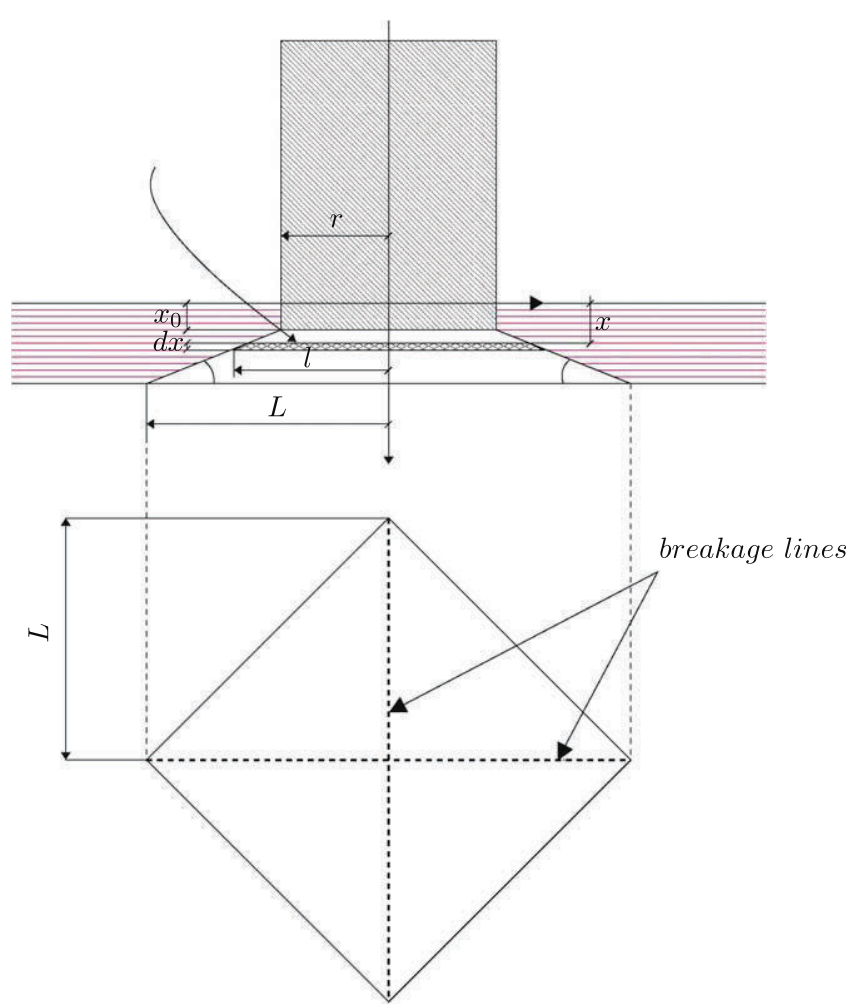

Fig. 4. Fiber failure breakage scheme. 
$c_{r}(x) \begin{cases}0 & \text { if } 0<x<x_{0} \\ 1 & \text { if } x_{0}<x<h \\ 0 & \text { if } x \geqslant h\end{cases}$

The value of $x_{0}$ could be determined as follows:

$\int_{0}^{x_{0}} \frac{d \chi}{v(\chi)} \frac{2 h}{\sqrt{ } E_{3} / \rho}$

where $\chi$ is a displacement dummy variable.

- Energy absorbed by laminate crushing: the projectile breaks the laminate by compression. In a differential displacement $d x$, the energy associated with this mechanism is evaluated as the prod uct of the through thickness compressive strength of the lami nate $\sigma_{c}(x)$, that varies along the thickness, the projectile frontal area $A$ (which is constant for a cylinder), and the distance covered by the bullet $d x$. This term is written as follows:

$$
d E_{c}(x) \quad \sigma_{c}(x) A d x
$$

This energy absorption mechanism disappears when the pene tration process finishes, for $x>h$. The definition of the function $\sigma_{c}(x)$, is described in the following section.

\section{Estimation of the through-thickness compressive strength}

In order to quantify the value of the compressive strength of the laminate in the through thickness direction, quasi static punch shear tests were accomplished. Carbon/epoxy laminates shows very little strain sensitivity [33], and in this work it is assumed that it does not affect in the through thickness compressive strength. These test were performed on a Universal Testing Machine, where the puncture on the laminate sample was applied through a $5.5 \mathrm{~mm}$ diameter cylinder with flat tip, that presents identical diameter to that of the projectile used in the ballistic tests. Fig. 5 shows a sketch of the configuration used. During the test, the dis placement of the upper free surface of the laminate, $\delta_{f}$, is measured using an Instron LVDT extensometer located far from the punch cylinder. Taking into account the stiffness of the cylinder, obtained by a compression test of the tool, the displacement at the cylinder specimen contact surface, $\delta_{t}$, can be determined during the punch shear test, and therefore, the relative displacement could deter mine the real depth of penetration $\delta$ on the laminate.

Two different types of tests were accomplished:

- Crushing: at the initial instants of the penetration process, for times $t<t_{0}$, it can be considered that the projectile impacts on a semi infinite body. Then, the laminate compressive strength can be obtained by placing a steel plate in the bottom face of the laminate (Fig. 6a shows the set up).
- Punching: for times $t \geqslant t_{0}$, the stress wave is already propagated along the whole laminate thickness, and then the semi infinite body assumption is not longer valid. In this situation it is consid ered that the bottom laminate face is free, and then the steel plate placed in contact with the bottom face of the specimen presents a hole with a diameter of $20 \mathrm{~mm}$ (Fig. 6b). The size of the hole was estimated taking into account the affected area during the impact process, that is related to the one estimated for the fiber failure mechanism.

It was considered that only crushing process appears in these tests. The stress displacement curves obtained by these tests are shown in Fig. 7. In both cases the curves start growing quadrati cally, with higher slope in the punching case; later an approxi mately linear relation is observed. In order to define the value of the compressive strength of the laminate $\sigma_{c}(x)$ both curves were fitted by linear and parabolic regressions. Using this polynomial functions defined by parts as function of the deep of penetration $(x)$, it is possible to obtain an analytical solution of the differential equation. This result is shown in the next section.

\section{Equation resolution}

Once the four differential energy terms are described, and the compressive strength is defined, the equation for the obtaining of the velocity could be defined:

$$
\begin{aligned}
\frac{1}{2} m_{p} d\left(v^{2}(x)\right) \quad & \sigma_{c}(x) A d x+\frac{1}{2}\left[A d x \rho_{l}\right] v^{2}(x) \\
& +2 d x \varepsilon_{f} X_{t}\left(r+\left(\begin{array}{ll}
x & x_{0}
\end{array}\right) \tan \alpha\right)^{2} c_{r}(x)
\end{aligned}
$$

Rearranging the terms and making some simplifications it is possible to rewrite the differential equation of the problem, with its initial condition:

$$
\left\{\begin{array}{c}
\frac{1}{2} m_{p} \frac{d v^{2}(x)}{d x} \\
v(0) \quad v_{i}
\end{array} \quad \sigma_{c}(x) A+\frac{1}{2}\left(A \rho_{l}\right) v^{2}(x)+2 \varepsilon_{f} X_{t}\left(r+\left(\begin{array}{ll}
x & x_{0}
\end{array}\right) \tan \alpha\right)^{2} c_{r}(x)\right.
$$

In order to understand the influence of each parameter, dimensionless variables are introduced. The velocity and the po sition of the projectile are dimensionalized with initial velocity and specimen thickness, respectively $\left(v^{*} \quad v / v_{i}\right.$ and $\left.x^{*} x / h\right)$. The compressive strength is redefined as $\sigma_{c}^{*}\left(x^{*}\right) \quad \sigma_{c}(x) / \sigma_{0}$ (where $\sigma_{0}$ is the strength in the through thickness direction, sup plied by the manufacturer). In addition a change of variable for the velocity is introduced to simplify the resolution of the equa tion: $w^{*} \quad v^{* 2}$. Finally the dimensionless differential equation could be written as:

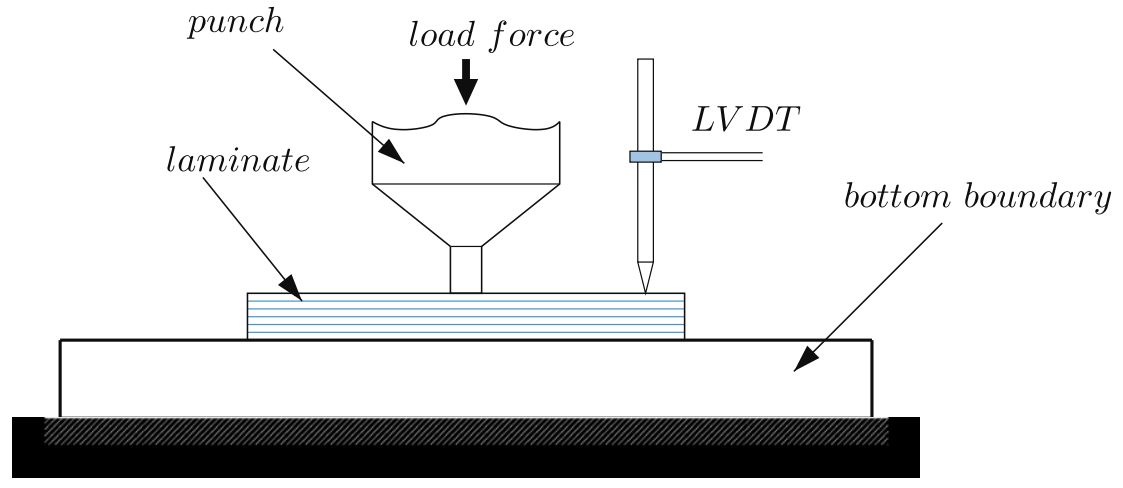

Fig. 5. Scheme of the set-up. 
(a)

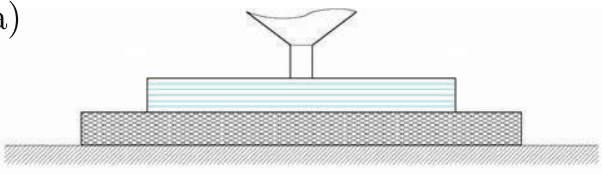

(b)

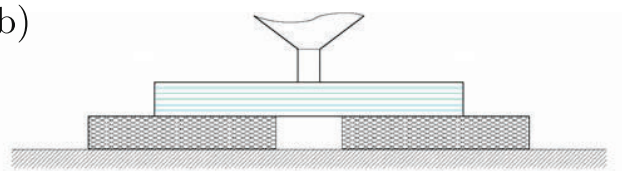

Fig. 6. Boundary conditions: (a) crushing and (b) punching.
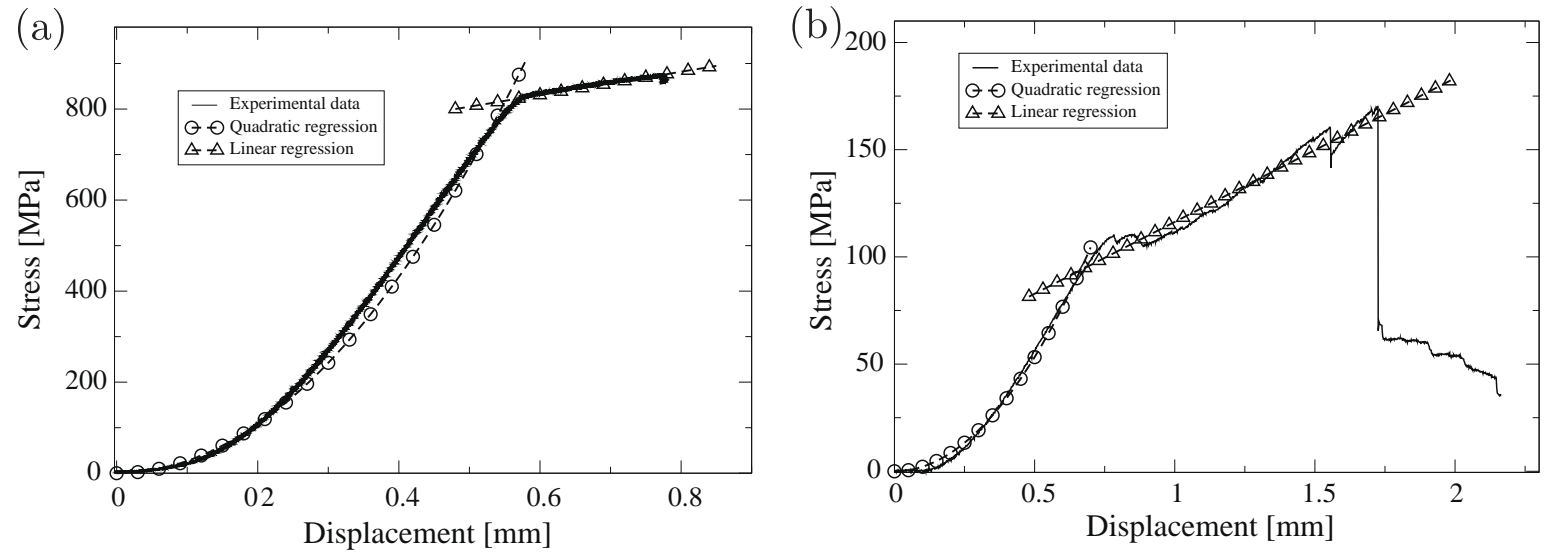

Fig. 7. Experimental data obtained for both boundary conditions: (a) crushing and (b) punching.

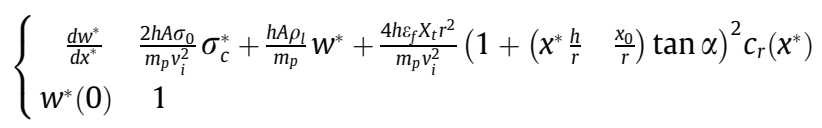

The following equations collect the constants that multiply each term of the differential equation and its order of magnitude. These constants are defined in order to analyze its relevance.

$$
\begin{array}{ll}
R_{c k} \quad \frac{2 h \sigma_{0} A}{m_{p} v_{i}^{2}} \sim \frac{5 \times 10^{3}}{v_{i}^{2}} \\
R_{m} \quad \frac{h A \rho_{l}}{m_{p}} \sim 10^{2} \\
R_{f k} \quad \frac{4 h \varepsilon_{f} X_{t} r^{2}}{m_{p} v_{i}^{2}} \sim \frac{10^{3}}{v_{i}^{2}}
\end{array}
$$

The first term $R_{c k}$ represents the ratio between the energy ab sorbed by crushing, and the kinetic energy of the projectile; $R_{m}$ is the ratio between the mass of the laminate (affected by the im pact), and the mass of the projectile; the last term $R_{f k}$ is related to the ratio between the energy absorbed by the tensile failure of fibers and, once again, the kinetic energy of the projectile.

Fig. 8 shows the value of the three aforementioned dimension less ratios as a function of the impact velocity of the projectile. At low impact velocities the term which represents the ratio between crushing energy dissipation and the projectile kinetic energy $\left(R_{c k}\right)$ is the most important, while at high velocities the mass ratio term $\left(R_{m}\right)$ is the most important. This analysis allows to state that at low impact velocities the kinetic projectile energy is mainly absorbed by laminate crushing, while at high velocities the linear momen tum transfer drives the process [34].

Finally the equation that must be solved is the following, where asterisk are omitted for simplicity:

$$
\left\{\begin{aligned}
\frac{d w}{d x} & R_{c k} \sigma_{c}+R_{m} w+R_{f k}\left(1+\left(x_{\frac{h}{r}} \frac{x_{0}}{r}\right) \tan \alpha\right)^{2} c_{r}(x) \\
w(0) & 1
\end{aligned}\right.
$$

which has an exact solution, in the form of: $w(x) \quad c_{1} \exp \left(R_{m} x\right)+c_{2} x^{2}+c_{3} x+c_{4}$

Due to the mathematic complexity of this solution, mainly be cause of the strength in the through thickness direction is de fined by parts, a numerical integration is used to calculate the results.

\section{Experimental tests for model validation}

In order to validate the analytical model, experimental tests were accomplished on carbon/epoxy laminates with 10 plies, $\left([0]_{10}\right)$, and a total thickness of $2.2 \mathrm{~mm}$. The specimen size is $80 \times 80 \mathrm{~mm}^{2}$. The projectile launched against the plate is a tem pered steel cylinder with $5.5 \mathrm{~mm}$ diameter and $6.0 \mathrm{~mm}$ length, being its mass $1.1 \mathrm{~g}$. The tempered steel is hard enough to ensure that no plastic deformation will occur during penetration, thus simplifying the analysis because all the energy associated to the projectile is kinetic.

The launcher employed in the impact tests is a single stage light gas gun which uses a helium gas bottle at a pressure of 200 bar to impel the projectile up to $500 \mathrm{~m} / \mathrm{s}$ (Fig. 9). The projectile travels through a gallery in which two photoelectric cells detect the pass ing of the projectile, allowing to obtain the impact velocity. At the end of the gallery, the projectile reaches an armored chamber $\left(1 \times 1 \times 1 \mathrm{~m}^{3}\right)$ where the specimen is placed by means of a support which ensures the appropriate position and gripping of the speci men. The chamber has two windows, one in a lateral side and an other on the top, that allow to capture the video sequence of the impact (Fig. 10).

To measure the residual velocity, a Photron Ultima APX digital high speed camera was employed. The selected frame rate was 15,000 per second, so that a picture is taken every $67 \mu$ s. The res olution was $1024 \times 128$ pixels and the shutter was set to $11 \mu \mathrm{s}$. These settings were selected based on early testing and represent an optimal trade off between available lighting and the minimiza tion of blur in the images. The camera was placed on the top of the chamber so that the entry and the exit of the projectile is perfectly 


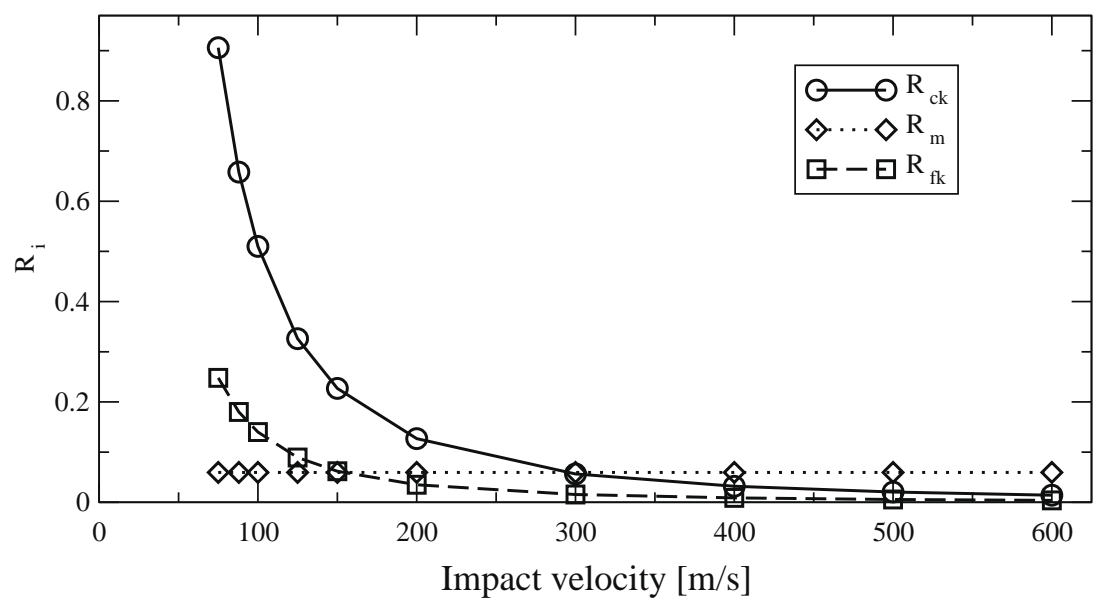

Fig. 8. $R_{c k}, R_{f k}$ and $R_{m}$ as function of impact velocity.

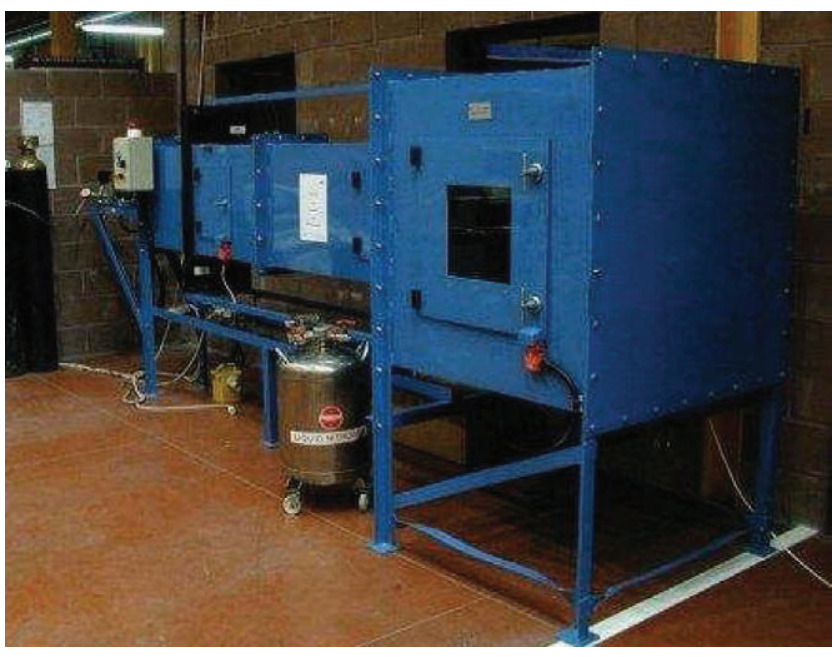

Fig. 9. Gas gun experimental device.

captured. A sequence recorded by the high speed camera is shown in Fig. 10.

\section{Results}

\subsection{Validation}

To validate the proposed model, impacts at velocities between 100 and $400 \mathrm{~m} / \mathrm{s}$ were performed. This wide range of velocities al lows to perform the validation above and below the ballistic limit. Fig. 11 shows the residual velocity of the projectile for different im pact velocities; experimental and analytical results are compared. The model predicts with high accuracy the residual velocity and in addition estimates the minimum impact velocity of the projec tile needed to perforate the laminate, which is around $130 \mathrm{~m} / \mathrm{s}$ for this combination of laminate and projectile. As the impact velocity increases, the curve tends to a straight line, which is the expected result.

\subsection{Analysis of the energy absorption mechanisms}

Fig. 12 shows the relative importance of each absorption mech anism as a function of the impact velocity. As the impact velocity increases, the term related to the linear momentum transfer increases its relative importance, reaching the $60 \%$ of the total en

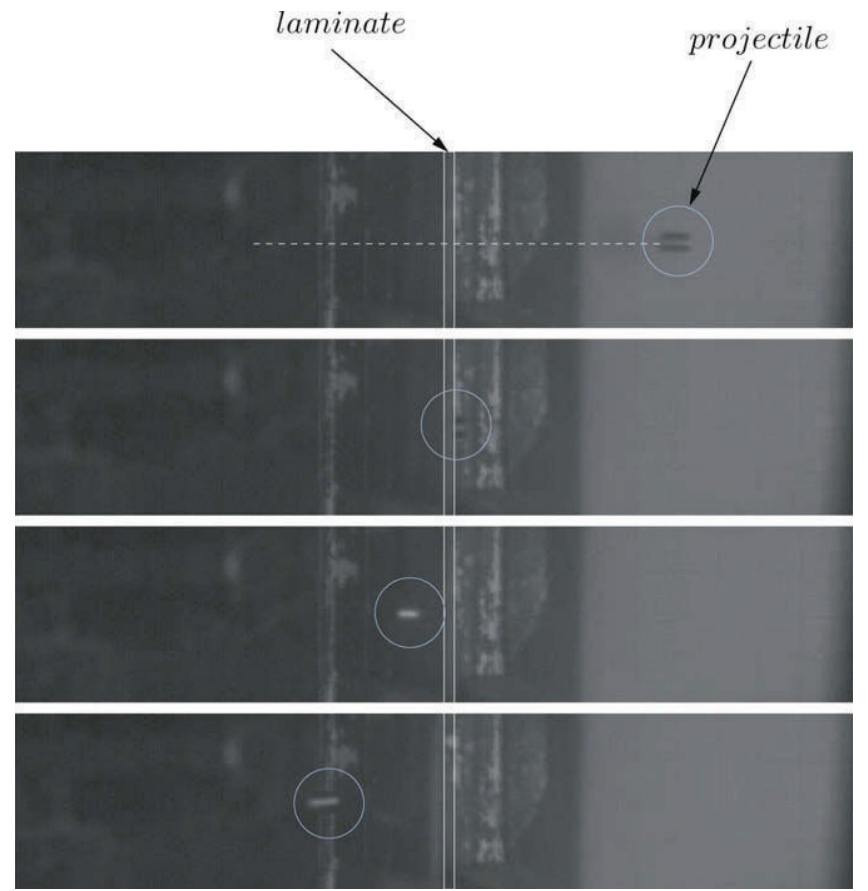

Fig. 10. Sequence of an impact process

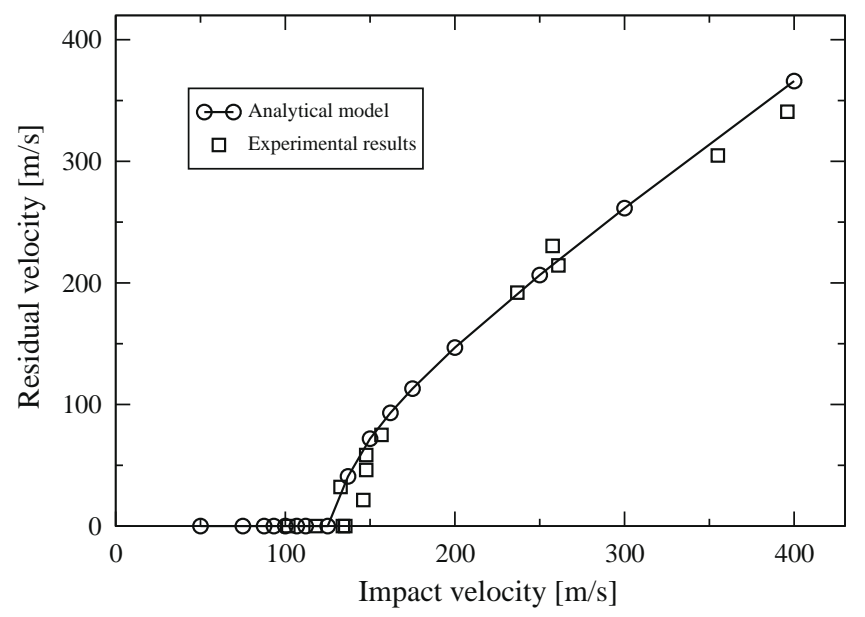

Fig. 11. Residual velocity vs. impact velocity; analytical and experimental results. 


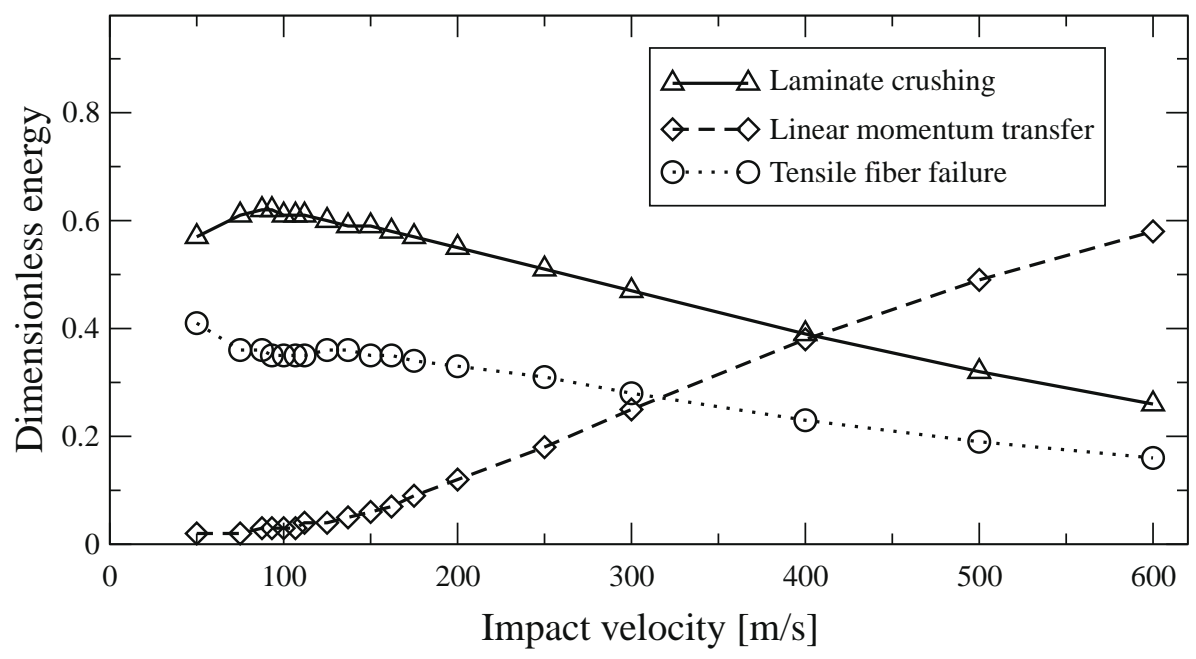

Fig. 12. Dimensionless energy vs. impact velocity.

ergy absorbed by the laminate when the projectile impact velocity is $600 \mathrm{~m} / \mathrm{s}$. The trend of the other two energy absorption mecha nisms (tensile fiber failure and laminate crushing) is almost the same for velocities above the ballistic limit (around $130 \mathrm{~m} / \mathrm{s}$ ): its importance decreases as the impact velocity increases because both mechanisms do not vary significantively with impact velocity. However, below ballistic limit its trends are different. Laminate crushing increases with impact velocity because more thickness of the laminate is involved in the penetration process. Tensile fiber failure decreases, because it starts to play a role only after $t t_{0}$; then, at low velocities this mechanism initiates when the projectile is closer to the impact face and involves more plies of the laminate.

\subsection{Influence of the projectile size}

In this section, an analysis of the influence of the projectile as pect ratio in the ballistic behavior of the laminate is presented. Fig. 13 shows the residual velocity vs. impact velocity for different projectile masses, keeping the radius constant. This variation rep resents a change in the density or in the length of the projectile. As $m_{p}$ increases the ballistic limit decreases and the curve tends to an asymptote which is the graph bisector. When the projectile mass tends to infinity, keeping the thickness and the density of the laminate constant, the ratio $R_{m}$ tends to zero and the residual velocity equals the impact velocity. In Fig. 13, the last curve corre

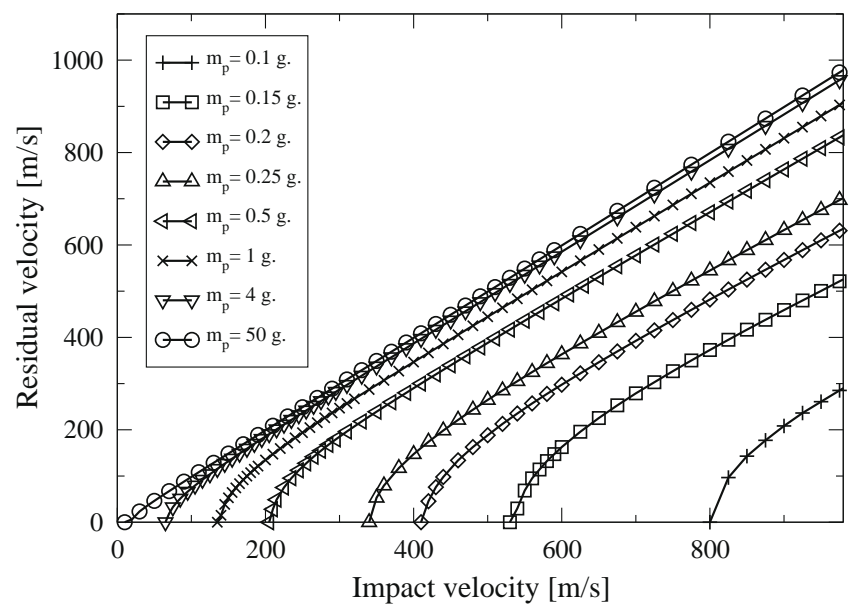

Fig. 13. Residual velocity vs. impact velocity, for different projectile masses; constant radius. sponds to $m_{p} 50 \mathrm{~g}$, which is three orders of magnitude greater than the laminate mass affected by the impact $\left(\rho_{l} A h\right)$.

Fig. 14 shows the evolution of the ballistic limit, as a function of the projectile mass keeping constant its radius. The curve has two

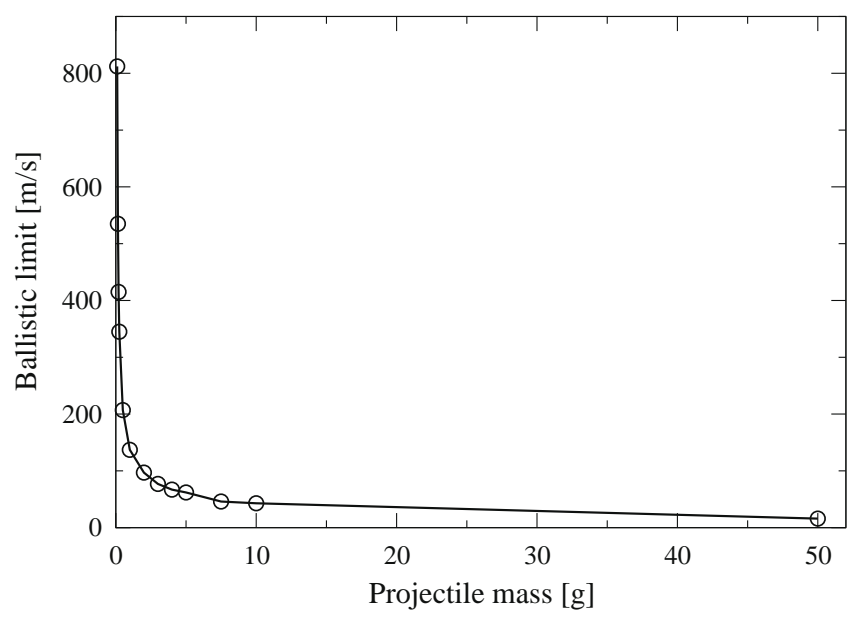

Fig. 14. Ballistic limit vs. projectile mass; constant radius.

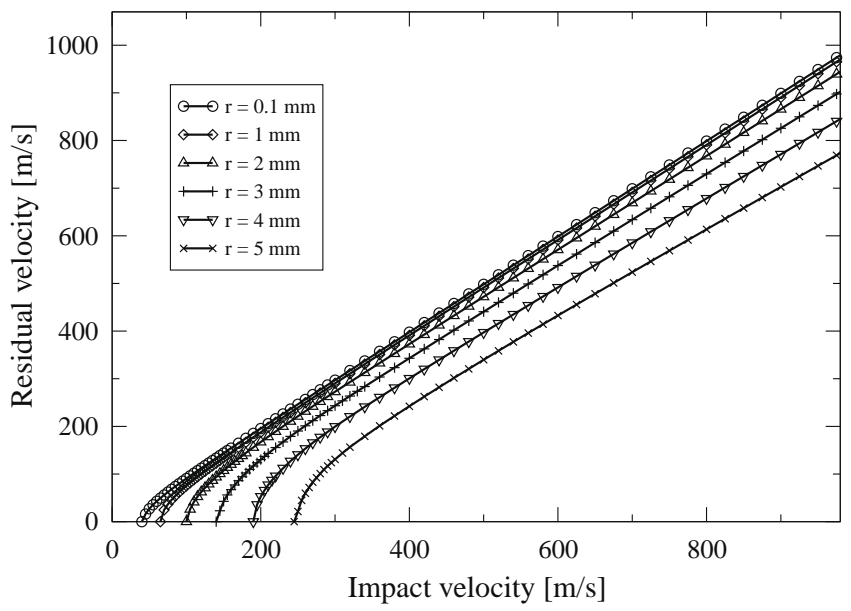

Fig. 15. Residual velocity vs. impact velocity, for different projectile radius; constant mass. 
asymptotes: the abscissa and the ordinate. When the projectile mass tends to infinity the ballistic limit tends to zero, and when the mass tends to zero, the impact velocity needed to penetrate the laminate tends to infinity. This graph shows the prediction capability of the developed model, and demonstrates that it pro vides expected tendencies in a wide range of projectile masses.

Finally Fig. 15 presents the residual velocity vs. impact velocity for different projectile radii keeping the mass constant; this varia tions means (as for Fig. 13) that the density of the projectile or its length is modified. As the radius decreases the curve tends to an asymptote which is the graph bisector (residual velocity equal to impact velocity), since the laminate zone affected by the impact is proportional to the frontal projectile area. As the radius increases the curve moves to the right, the ballistic limit also increases, and the slope corresponding to the high velocity regime decreases.

\section{Conclusions}

An analytical model to predict the residual velocity of cylindri cal projectiles after impacting thin carbon/epoxy woven laminates has been developed. The model considers three different energy absorption mechanisms for the laminate. As a summary of this work, the following conclusions could be found:

- The analytical model developed, predicts with accuracy the residual velocity of steel cylinder projectiles when penetrating carbon/epoxy woven laminates.

- The use of quasi static test (punch and crushing) to obtain a reli able value of the through thickness compressive strength of the laminate as a function of the depth of penetration is appropriate to estimate the energy absorbed by the composite by laminate crushing.

- Using the analytical model developed in this work as a pre design tool, it is possible to obtain trustworthy results of the influences of different problem parameters, such as projectile geometry or material.

\section{Acknowledgment}

This research was done with the financial support of the University Carlos III of Madrid and of the Comunidad Autónoma de Madrid under Projects CCG08 UC3M/MAT 4464 and CCG08 UC3M/DPI 4348.

\section{References}

[1] Tennyson RC. Composites in space - challenges and opportunities. Proc ICCM10 1995;1:35-56.

[2] David-West OS, Nash DH, Banks WM. An experimental study of damage accumulation in balanced CFRP laminates due to repeated impact. Compos Struct 2008;83:247-58.

[3] Airoldi A, Cacchione B. Modelling of impact forces and pressures in Lagrangian bird strike analyses. Int J Impact Eng 2006;32:1651-77.

[4] Anghileri M, Castelleti LML, Invernizzi F, Mascheroni M. A survey of numerical models for hail impact analysis using explicit finite element codes. Int J Impact Eng 2005;31:929-44.

[5] Mines RAW, McKown S, Birch RS. Impact of aircraft rubber tyre fragments on aluminium alloy plates: I-experimental. Int J Impact Eng 2007;34:627-46.
[6] Varas D, López-Puente J, Zaera R. Experimental analysis of fluid-filled aluminium tubes subjected to high-velocity impact. Int J Impact Eng 2009;36:81-91.

[7] Varas D, Zaera R, López-Puente J. Numerical modelling of the hydrodynamic ram phenomenon. Int J Impact Eng 2009;36:363-74.

[8] Hai-jun X, Rong-ren W. Aeroengine turbine blade containment tests using high-speed rotor spin test facility. Aerospace Sci Technol 2006;10:501-8.

[9] Schonberg WP. Hole size and crack length models for spacecrafts walls under oblique hypervelocity projectile impact. Aerospace Sci Technol 1999;3:461-71.

[10] Farenthold EP, Hernandez RJ. Simulation of orbital debris impact on the space shuttle wing leading edge. Int J Impact Eng 2006;33:231-43.

[11] Cantwell WJ, Morton J. Comparison of low and high velocity impact response of CFRP. Composites 1989;20(6):545-51.

[12] Sun CT, Potti V. A simple model to predict residual velocities of thick composite laminates subjected to high velocity impact. Int J Impact Eng 1996;18(3):339-53.

[13] Larsson F. Damage tolerance of a stitched carbon/epoxy laminate. Composites Part A: Appl Sci Manufact 1997;28:923-34

[14] Bland PW, Dear JP. Observations on the impact behaviour of carbon-fibre reinforced polymers for the qualitative validation of models. Composites Part A: Appl Sci Manufact 2001;32:1217-27.

[15] López-Puente J, Zaera R, Navarro C. The effect of low temperatures on the intermediate and high velocity impact response of CFRP. Composites: Part B 2002;33:559-66.

[16] López-Puente J, Zaera R, Navarro C. Experimental and numerical analysis of normal and oblique ballistic impacts on thin carbon/epoxy woven laminates. Composites: Part A 2008;39:374-87.

[17] Will MA, Franz T, Nurick GN. The effect of laminate stacking sequence of CFRP filament wound tubes subjected to projectile impact. Compos Struct 2002;58:259-70.

[18] Tanabe Y, Aoki M, Fujii K, Kasano H, Yasuda E. Fracture behavior of CFRPs impacted by relatively high-velocity steel sphere. Int J Impact Eng 2003;28:627-42.

[19] Tanabe Y, Aoki M. Stress and strain measurements in carbon-related materials impacted by a high-velocity steel sphere. Int J Impact Eng 2003;28:1045-59.

[20] Hammond RI, Proud WG, Goldrein HT, Field JE. High-resolution optical study of the impact of carbon-fibre reinforced polymers with different lay-ups. Int J Impact Eng 2004;30:69-86.

[21] Hosur MV, Vaidya UK, Ulven C, Jeelani S. Performance of stitched/unstitched woven carbon/epoxy composites under high velocity impact loading. Compos Struct 2004;64:455-66.

[22] Herzsberg I, Weller T. Impact damage resistance of buckled carbon/epoxy panels. Compos Struct 2006;73:130-7.

[23] Caprino G, Lopresto V, Santoro D. Ballistic impact behaviour of stitched graphite/epoxy laminates. Compos Sci Technol 2007;67:325-35.

[24] Hazell PJ, Kister G, Stennett C, Bourque P, Cooper G. Normal and oblique penetration of woven CFRP laminates by a high velocity steel sphere. Composites: Part A 2008;39:866-74.

[25] Kim H, Welch DA, Kedward KT. Experimental investigation of high velocity ice impacts on woven carbon/epoxy composite panels. Composites Part A: Appl Sci Manufact 2003;34:25-41.

[26] Chambers AR, Mowlem MC, Dokos L. Evaluating impact damage in CFRP using fibre optic sensors. Compos Sci Technol 2007;67:1235-42.

[27] Asp LE, Juntikka R. High velocity impact on NCF reinforced composites. Compos Sci Technol 2009;69(9):1478-82.

[28] Mines RAW, Roach AM, Jones N. High velocity perforation behaviour of polymer composite laminates. Int J Impact Eng 1999;22:561-88.

[29] Chen JK, Allahdadi FA, Carney TC. High-velocity impact of graphite/epoxy composite laminates. Compos Sci Technol 1997;57:1369-79.

[30] López-Puente J, Zaera R, Navarro C. High energy impact on woven laminates. J Phys IV 2003:639-44

[31] Fernández-Fdz D, López-Puente J, Zaera R. Prediction of the behaviour of CFRPs against high-velocity impact of solids employing an artificial neural network methodology. Composites: Part A 2008;39:989-96.

[32] López-Puente J, Zaera R, Navarro C. An analytical model for high velocity impacts on thin CFRPs woven laminates. Int J Solids Struct 2007;44:2837-51.

[33] Schoeppner GA, Abrate S. Delamination threshold loads for low velocity impact on composite laminates. Composites: Part A 2000;431:903-15.

[34] Hazell PJ, Cowie A, Kister G, Stennett C, Cooper GA. Penetration of a woven CFRP laminate by a high velocity steel sphere impacting at velocities of up to 1875 m/s. Int J Impact Eng 2009;36(9):1136-42. 